\title{
Development of a provisional essential medicines list for children in Canada: consensus process
}

\author{
Hannah Woods MSc, Efosa Oronsaye MBBS MPH, Anjli Bali MD, Yathavan Rajakulasingam MD, \\ Taehoon Lee MD MPH, Norman Umali RPh, Eyal Cohen MD MSc, Yaron Finkelstein BMedSc MD, \\ Martin Offringa MD PhD, Nav Persaud MD MSc
}

\section{Abstract}

Background: Worldwide, many countries have developed a list of essential medicines for children to improve prescribing. We aimed to create an essential medicines list for children in Canada.

Methods: We adapted the previously created preliminary list of essential medicines for adults in Canada and the WHO Model List of Essential Medicines for Children to create a provisional list of essential medicines for children in Canada. Canadian clinicians made suggestions for changes. Literature relevant to each suggestion was presented to clinician-scientists, who used a modified nominal group technique to make recommendations on the suggestions. Ontario Public Drug Programs prescription data were reviewed to identify commonly prescribed medications missing from the list. Literature relevant to these medications was shared with a clinicianscientist review panel to determine which should be added, and a revised list was developed.

Results: A total of 76 items were removed from the list of essential medicines for adults in Canada because they were not indicated for use in children or were not relevant in the Canadian health care context; 7 medications were added to the child list based on Ontario Public Drugs Programs prescribing data and clinician-scientist review. Suggestions to add, remove or substitute medications were made by peer-reviewers and resulted in removal of 1 medication and replacement of 1 medication. The process produced a provisional list of 67 essential medications for children.

Interpretation: A provisional list of 67 essential medicines for children was created through a peer-reviewed, multistep process based on current clinical evidence, Canadian clinical practice guidelines and historical prescribing data. It is publicly posted at http:// cleanmeds.ca/. The list should be further developed based on wider input and should be continuously revised based on emerging evidence of the safety and effectiveness of these medicines in all pediatric age groups.

I: n 1977, the World Health Organization (WHO) created a model list of essential medicines that is updated every 2 years based on up-to-date evidence for efficacy, safety and tolerability. ${ }^{1,2}$ The WHO recommends that each country evaluate and adapt the list in order to create a list of essential medicines that is appropriate for its own environment. Previously, we adapted the WHO model list of essential medicines to create a list of essential medicines for adults in Canada. ${ }^{3}$ This list may contribute to improved quality of care in that a short list of essential medications may make it easier for clinicians to prescribe the most effective, safe and appropriate medication ${ }^{4-6}$ and more appropriate use of drugs. ${ }^{7,8}$

The WHO developed its first model list of essential medicines for children in 2007 in an effort to make safe and effective medicines as available for children as for adults. ${ }^{9,10}$ The current fifth edition lists medications deemed to be the most efficacious, safe and cost-effective for priority conditions and diseases. The WHO recommends that the list of essential medicines for children be adapted by countries according to local context and policy.

In Canada, there is no list or central source of information related to safety, efficacy and tolerability of medication forms and formulations for children. This, in combination with the large number of medications with unknown safety and efficacy profiles available for children in Canada, ${ }^{11-13}$ poses a challenge for clinicians treating children. An essential medicines list for

Competing interests: None declared.

Disclaimer: Nav Persaud is an associate editor for CMAJ Open and was not involved in the editorial decision-making process for this article.

This article has been peer reviewed.

Correspondence to: Nav Persaud, nav.persaud@utoronto.ca

CMAJ Open 2018. DOI:10.9778/cmajo.20170129 
children in Canada may contribute to improvements in quality of care while also generating cost savings. ${ }^{7,8,12,14,15}$

Our aim was to develop a list of essential medicines for children in Canada based on the WHO Model List of Essential Medicines for Children ${ }^{16}$ and on the adult essential medicines list we previously created. ${ }^{3}$

\section{Methods}

\section{Adaptation of World Health Organization essential medicines list}

As previously described, ${ }^{3}$ we adapted the 2013 WHO essential medicines list $^{16}$ to create a preliminary essential medicines list for adults in Canada. The purpose of this process was to identify the medicines on the WHO list that are applicable to Canada. Medicines were removed from the WHO list for 1 of 5 reasons: an item was not a medication, other medications on the list had better tolerated routes of administration (e.g., oral instead of intravenous), the medication had the same indication as other listed medications, the medication was used for conditions that are uncommon in Canada, or the medication was not a medication prescribed by primary care providers. Any disagreements were resolved through discussion and consensus. ${ }^{3}$

After reviewing the WHO list, we considered adding medications applicable to Canada that were not on the WHO list. We reviewed Canadian clinical practice guidelines, systematic reviews, health technology assessment reports and child formularies in other countries to determine whether there were medications applicable to Canada that were not on the WHO list. This part of the process generated a draft of the list for wider feedback.

\section{Development of the provisional list of essential medicines for children in Canada}

We started with medications that were on both the Canadian adult list ${ }^{3}$ and the WHO Model List of Essential Medicines for Children, 5th list. ${ }^{9}$ All medications on our adult essential medicines list that also appeared on the WHO model list for children were added to our draft essential medicines list for children. Medications that were on the WHO model list for children but not on our provisional list were identified for potential inclusion. For each of these medications, research team members determined whether there was an equivalent medication on the Canadian child list or whether the indication for which the medication was prescribed using RxTx (formerly e-CPS) or whether the medicine was not used in Canada (e.g., treatment for a tropical disease). If there was an equivalent, or if the medication was not relevant in the Canadian health care context, the medication was not added to our provisional list of essential medicines for children in Canada.

\section{Peer review feedback}

Peer reviewers included pediatricians, primary care physicians, nurse practitioners, pharmacists, and consultants or specialists practising in Canada. The initial child list of essential medicines was made publicly available via a website (www. cleanmeds.ca), and feedback on suggested changes to medica- tions on the list was collected through the website. Each proposed change was classified as a replacement, an addition to or a removal from the list and could be justified by at least 1 of the following: evidence of efficacy, evidence of safety, route of administration and tolerability, dosing schedule, usefulness for other medical conditions and interactions with other medications. Respondents were allowed to make suggestions for any reason. The website has been active since January 2017, and changes can be suggested at any time.

Peer reviewers were carefully selected based on expertise, publications and academic involvement. We searched Canadian clinical practice guidelines using the repository maintained by the Canadian Medical Association and publications in CMAf, Canadian Family Physician and Paediatrics \& Child Health in the previous 2 years for authors of papers in different pediatric therapeutic areas. Thirteen peer reviewers were contacted with a description of the project and the website (www.cleanmeds.ca) where they could submit their proposed list changes. Peer reviewers were first contacted by email, and, if we did not receive a response, we followed up by fax or telephone. Three attempts were made to contact peer reviewers.

Based on the suggested changes to the adapted list made by the peer reviewers, we developed questions focused on efficacy and safety with support from an information scientist and performed a literature search for each question. Five literature searches were performed, 1 for each suggestion that was made. No searches were run for medicines that remained from the WHO list. Duplicate or similar suggestions were grouped together in 1 question. Evidence was gathered from systematic reviews, meta-analyses, randomized controlled trials, the Compendium of Pharmaceuticals and Specialties, clinical practice guidelines and health technology assessment reports such as those from the Canadian Agency for Drugs and Technologies in Health. Members of the research team (A.B., E.O., H.W., Y.R. and N.P.) reviewed the results of the literature searches and compiled the information into an evidence report document. Literature search questions and search strategies were included in the evidence report and are publicly posted online (at cleanmeds.ca/list/suggest-changes/).

Five clinician-scientists who were all active researchers involved in the treatment of children were invited to join meetings to discuss the suggestions made by the peer reviewers. They were asked to participate based on their familiarity with clinical issues relevant to the medications on the list, their experience critically appraising clinical evidence (i.e., research training, experience) and a lack of relevant conflicts of interest (including those with the pharmaceutical industry). A meeting was held on Mar. 30, 2017, to discuss changes that were suggested by peer reviewers via the website. Three voting members (clinician-scientists) and one of the authors (N.P., nonvoting) were present at the meeting. Each participating clinician-scientist was given the evidence report to review 2 weeks before the meeting. Clinician-scientists used the information in the documents to form an evidence-based recommendation on addition, removal or replacement for each suggested medication change. The clinician-scientists submitted comments on each suggestion to the research team before the 
meeting. The comments were compiled by research assistants (A.B., T.L., E.O., Y.R. and H.W.) and presented to all clinician-scientists during the meeting to facilitate discussion among the clinician-scientists. Each voting member discussed his or her opinion without interruption, followed by open discussion. After each group discussion, the participating clinicianscientists voted by independently recommending whether the suggested change should be made based on the evidence gathered and from their own clinical expertise. The meeting used a modified nominal group technique, involving independent consideration before the meeting, group discussion and voting on recommended changes to the adapted list.,17

The strength of each recommendation (strong or weak) was determined by the 3 participating clinician-scientists. The final recommendations were deemed strong if all clinicianscientists were in agreement for or against the recommendation and at least 2 had made strong recommendations. If this criterion was not met, the recommendation was deemed weak in the direction of the majority of clinician-scientist votes. The strength of evidence supporting each recommendation was determined by vote using the Grading of Recommendations Assessment, Development and Evaluation (GRADE) system. ${ }^{3,18}$ The strength of the recommendation reflects the importance of the decision, whereas the strength of the evidence reflects how unlikely it is that new evidence would change the recommendation.

\section{Identification and addition of commonly prescribed medications}

To identify commonly prescribed medications missing from the list, we reviewed the Ontario Public Drug Programs prescription data. These programs provide publicly funded medications to social assistance recipients and others. For each medication, if there was an equivalent on the draft list of child essential medicines according to the Canadian RxTx, the medication was not selected for further review. Medications were considered equivalent if they treated the same condition and/ or were from the same class of medications. A physician (E.O.) and a pharmacist (N.U.) completed this process. The medications that were not already on the provisional list of essential medicines for children and for which there were no equivalents were selected for further review. Evidence reports presenting current information about the effectiveness and safety of these medications were created and disseminated to clinicianscientists for review. A clinician-scientist meeting was held on Mar. 23, 2017, at which the participants deliberated and voted for or against addition of each medication to the child essential medicines list. Three voting members (clinician-scientists) and N.P. were present at the meeting. Where there was consensus, the medication was added or not added to the list, as appropriate, and the website was updated. If consensus was not reached, the decision whether to add the medication was deferred until more evidence could be provided.

\section{Patient and community involvement}

The process for developing and revising the list was codeveloped with a panel of 11 community members recruited from the area surrounding St. Michael's Hospital, Toronto, through canvassing, random-digit dialing and existing community groups. The community guidance panel met every 1-2 months during the development of the list and provided input on issues including the criteria used to select medications, how to maintain the list and the knowledge translation strategy. The members of the community guidance panel did not suggest particular changes to the medications on the list.

\section{Ethics approval}

The study was approved by the Research Ethics Board of St. Michael's Hospital, Toronto.

\section{Results}

\section{Adaptation of essential medicines list for adults}

The most recent preliminary essential medicines list for Canada contains 134 items. ${ }^{3}$ In creating our provisional list for children, 73 items were removed from the adult list because they were not indicated for use in children in the WHO Model Formulary for Children or were not relevant in the Canadian health care context. This resulted in a provisional child list of 61 essential medi-

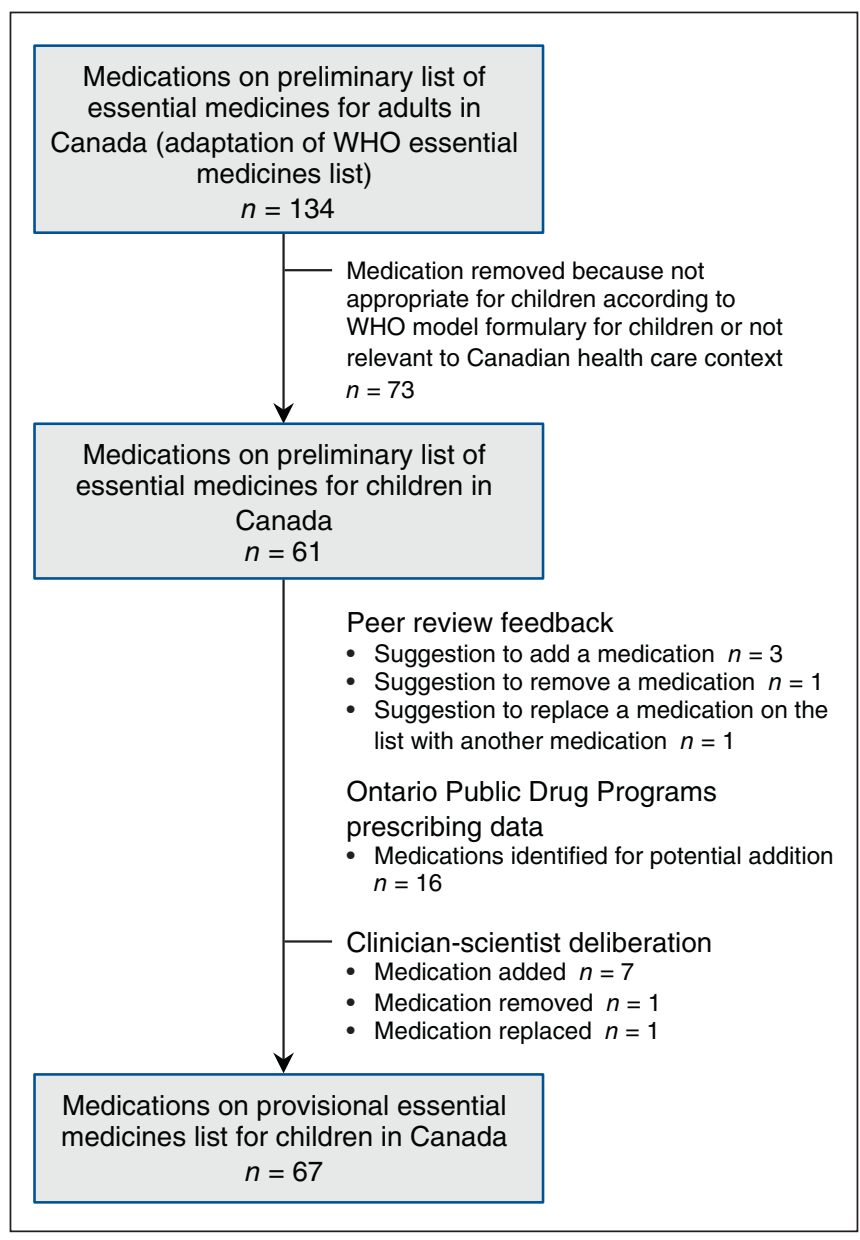

Figure 1: Flow diagram summarizing the development of the provisional essential medicines list for children in Canada. Note: WHO = World Health Organization. 
cines. Figure 1 illustrates the development process for the child list of essential medicines. The list can be found at cleanmeds.ca.

\section{Peer-review suggestions}

Four peer reviewers suggested changes to the list; the response rate was $31 \%$.

Three suggestions to add, 1 suggestion to remove and 1 suggestion to substitute medications on the list were made by peer-reviewers. Deliberations that took place at clinicianscientist meetings led to removal of 1 medication and replacement of 1 medication.

\section{Identification of commonly prescribed medicines}

Evidence reports for the 16 potential additions to the list based on Ontario Public Drug Programs prescribing data were presented to clinician-scientists for review, and a clinician-scientist meeting was held at which deliberation took place. As a result of this process, 7 medications were added to the essential medicines list for children in Canada.

\section{Clinician-scientist review}

All invited clinician-scientists agreed and participated; the response rate was $100 \%$. The suggested changes based on peer reviewers and commonly prescribed medications together resulted in a list of 67 medications (Appendix 1, available at www.cmajopen.ca/content/6/1/E146/suppl/DC1).

\section{Interpretation}

We adapted the provisional essential medicines list for adults using a 4-step process involving a small group of Canadian clinicians and clinician-scientists. The provisional essential medicines list for children in Canada contains 67 medications. This is a work in progress, and the list will likely be revised and grow as further input is gathered. The current short list may allow clinicians to learn more about fewer drugs and could improve appropriateness of clinician prescribing. ${ }^{3,19}$

Essential medicine lists for children differ from country to country in the way they are developed and presented; some countries include details of the list development process and guidelines for preparation, prescribing and how to use the document. Some lists are stand-alone documents specific to children, whereas others are a pediatric section within a larger list or formulary. The number of medications included in child essential medicine lists or formularies ranges from 4 (Egypt) to over 1000 (United Kingdom). ${ }^{20,21}$ List information by country is presented in Supplementary Table S1, Appendix 1.

Details on the development process for pediatric lists and formularies are not readily available for all countries; however, some countries make this information publicly available. The British National Formulary for Children and the Kinderformularium (Dutch Paediatric Formulary) present information on the development of their child formularies including the contributing bodies, the sources of information and how the information was validated. ${ }^{21,22}$ Although these are not essential medicines lists, their purpose is similar to that of our child essential medicines list: to aid decisions on prescribing, dis- pensing and administering medicines. ${ }^{21,22}$ The process for developing the British National Formulary for Children and the Dutch Paediatric Formulary is aligned with the process for developing our child essential medicines list: collaboration; consulting expert clinical advisors, the literature, systematic reviews, consensus guidelines, reference sources and comments from readers; and continuous revision are important components. ${ }^{23}$ The British National Formulary for Children also includes information in such areas as how to use the formulary, selecting suitable preparations, dosage selection and writing prescriptions. ${ }^{21}$ Likewise, South Africa includes these details around the development process for their Standard Treatment Guidelines and Essential Medicines List for hospital-level pediatrics, as does India for their Essential Medicines List for Children of India. ${ }^{24,25}$ The processes and methods used in other jurisdictions will continue to be consulted as we further develop and maintain the provisional essential medicines list for children in Canada.

The provisional list of essential medicines for children we have created lists medications in alphabetical order or therapeutic area and includes the source of the suggestion. Further details and the presentation of our list for children are to be decided through additional input and collaboration.

\section{Limitations}

The development process was dependent on evidence for the individual medicines, and high-quality evidence in children was often lacking. It is unclear whether it would be better to consider lower-quality studies. More high-quality evidence would facilitate development of the child essential medicines list. In many cases, there is not a clear basis for prescribing advice in children. We reviewed only the evidence for suggested changes; some medications on the WHO model list of essential medicines may not be well studied in children despite reviews conducted by that organization. The list may include medicines from the WHO model list that are not effective or are harmful.

The list was peer reviewed by a small number of people. Input through the website could be increased by direct advertising of the website to a range of clinicians. Other medications, including those usually prescribed by subspecialists (e.g., seizure disorder medications), may have been suggested had there been more peer reviewers. In the future, reviewers should include subspecialists such as pediatric neurologists. The small number of clinician-scientists making the final decisions meant that the final composition of the list may have been dependent on the judgments of just a few people.

Using commonly prescribed medications as one of the starting points for list development may have reinforced inappropriate prescribing practices; however, we created evidence reports regarding safety and efficacy for each medication and disseminated them to clinician-scientists before final recommendations on whether to add these medications to the list. Also, there are local and systems processes that can address inappropriate prescribing that occurs today without a list.

We did not consider local availability of medications in creating the provisional essential medicines list for children in 
Canada; the current availability of medicines as products that can be ordered through Canadian pharmacy wholesalers was not within the scope of this research.

\section{Conclusion}

We have developed a provisional short list of essential medications for children in Canada that can be refined in the future based on wider input. The list should be continuously revised by peer reviewers and clinician-scientists based on new evidence. Future work should determine the applicability of the list across Canada, the impact of list adoption on actual prescribing and the effects of list-driven prescribing on patients.

\section{References}

1. Essential medicines selection: National Medicines List/formulary/standard treatment guidelines. Geneva: World Health Organization; 2014. Available: www.who.int/selection_medicines/country_lists/en/ (accessed 2018 Mar. 16).

2. The selection of essential drugs: report of a WHO Expert Committee. WHO Technical Report Series, no. 615. Geneva: World Health Organization;1977.

3. Taglione MS, Ahmad H, Slater M, et al. Development of a preliminary essential medicines list for Canada. CMAJ Open 2017;5:E137.

4. Yakabowich MR, Keeley G, Montgomery PR. Impact of a formulary on personal care homes in Manitoba. CMA7 1994;150:1601-7.

5. Feely J, Chan R, Cocoman L, et al. Hospital formularies: need for continuous intervention. BM7 1990;300:28-30.

6. King MA, Roberts MS. The influence of the Pharmaceutical Benefits Scheme (PBS) on inappropriate prescribing in Australian nursing homes. Pharm World Sci 2007;29:39-42.

7. Eom G, Grootendorst P, Duffin J. The case for an essential medicines list for Canada. CMA7 2016;188:E499-503.

8. Sketris I, Ingram EL, Lummis H. Optimal prescribing and medication use in Canada: challenges and opportunities. Toronto: Health Council of Canada; 2007.

9. WHO model list of essential medicines for children. 5th list. Geneva: World Health Organization; 2015. Available: www.who.int/medicines/publications/ essentialmedicines/en/index.html (accessed 2018 Mar. 16).

10. Better medicines for children. Sixtieth World Health Assembly: resolution WHA60.20. Geneva: World Health Organization; 2007. Available: www.who. int/entity/childmedicines/publications/WHA6020.pdf (accessed 2018 Mar. 16).

11. Ontario Public Drug Programs. Formulary: drugs funded by Ontario Drug Benefit (ODB) Program. Toronto: Ministry of Health and Long-Term Care; 2014. Available: www.health.gov.on.ca/en/pro/programs/drugs/odbf_mn.aspx (accessed 2018 Mar. 16).

12. Alberta Drug Benefit List (ABDL). Edmonton: Alberta Health; 2015. Available: www.health.alberta.ca/services/drug-benefit-list.html (accessed 2018 Mar. 16).

13. Prescription drug insurance. Prescription drugs covered: prescription drugs covered by the Public Prescription Drug Insurance Plan. Québec: Régie de l'assurance maladie du Québec; 2015. Available: www.ramq.gouv.qc.ca/en/ citizens/prescription-drug-insurance/pages/prescription-drugs-covered.aspx (accessed 2018 Mar. 16).

14. Quittner AL, Modi AC, Lemanek KL, et al. Evidence-based assessment of adherence to medical treatments in pediatric psychology. 7 Pediatr Psychol 2008; 33:916-36.
15. Expert Panel on Therapeutic Products for Infants, Children, and Youth. Improving medicines for children in Canada. Ottawa: Council of Canadian Academics; 2014. Available: www.scienceadvice.ca/uploads/eng/assessments\%20 and $\% 20$ publications $\% 20$ and $\% 20$ news\%20releases/therapeutics/therapeutics_ fullreporten.pdf (accessed 2018 Mar. 16).

16. WHO model list of essential medicines. 18th list. Geneva: World Health Organization; 2013. Available: www.who.int/medicines/publications/ essentialmedicines/18th_EML_Final_web_8Jul13.pdf (accessed 2018 Mar. 16).

17. Nair R, Aggarwal R, Khanna D. Methods of formal consensus in classification/ diagnostic criteria and guideline development. Semin Artbritis Rheum 2011;41: 95-105.

18. Guyatt GH, Oxman AD, Vist GE, et al. GRADE: an emerging consensus on rating quality of evidence and strength of recommendations. BMF 2008;336:924-6.

19. Avery AJ, Walker B, Heron T, et al. Do prescribing formularies help GPs prescribe from a narrower range of drugs? A controlled trial of the introduction of prescribing formularies for NSAIDs. Br 7 Gen Pract 1997;47:810-4.

20. Egyptian National Formulary. Egypt: Central Administration of Pharmaceutical Affairs, Ministry of Health and Population; 2007. Available: http://apps.who. int/medicinedocs/documents/s17411e/s17411e.pdf (accessed 2018 Mar. 16).

21. Paediatric Formulary Committee. British National Formulary for Children (2015). London (UK): BM7 Group; 2015

22. van der Zanden TM, de Wildt SN, Liem Y, et al. Developing a paediatric drug formulary for the Netherlands. Arch Dis Child 2017;102:357-61.

23. Kelly LE, Ito S, Woods D, et al. A comprehensive list of items to be included on a pediatric drug monograph. 7 Pediatr Pharmacol Ther 2017;22:48-59.

24. Standard Treatment Guidelines and Essential Medicines List for South Africa. 2013 edition. Pretoria (South Africa): National Department of Health; 2013. Available: www.kznhealth.gov.za/pharmacy/PaedsSTG2013LR.pdf (accessed 2018 Mar. 16).

25. Indian Academy of Pediatrics List of Essential Medicines for Children of India. First list - 2011. Mumbai: Indian Academy of Pediatrics; 2011. Available: http://apps.who.int/medicinedocs/documents/s19040en/s19040en.pdf (accessed 2018 Mar. 16).

Affiliations: Centre for Urban Health Solutions (Woods, Oronsaye, Bali, Rajakulasingam, Lee, Umali, Persaud), Li Ka Shing Knowledge Institute, St. Michael's Hospital; Department of Family and Community Medicine (Persaud), University of Toronto; The Hospital for Sick Children (Cohen, Finkelstein, Offringa); University of Toronto (Cohen, Finkelstein, Offringa); Institute of Health Policy, Management and Evaluation (Cohen, Persaud), Toronto, Ont.

Contributions: Nav Persaud conceived of and designed the study. Nav Persaud, Hannah Woods, Efosa Oronsaye, Anjli Bali, Yathavan Rajakulasingam and Taehoon Lee acquired the data. Hannah Woods and Nav Persaud drafted the manuscript, and Nav Persaud, Hannah Woods, Efosa Oronsaye, Anjli Bali, Yathavan Rajakulasingam, Taehoon Lee, Eyal Cohen, Yaron Finkelstein and Martin Offringa revised it critically for important intellectual content. All of the authors analyzed and interpreted the data, gave final approval of the version to be published and agreed to be accountable for all aspects of the work.

Acknowledgement: The authors thank Lee Dupuis for helpful comments on the article.

Supplemental information: For reviewer comments and the original submission of this manuscript, please see www.cmajopen.ca/content/6/1/ E146/suppl/DC1. 